\title{
Urinary nerve growth 53 factor correlates with the severity of urgency and pain: response to comments by Agilli et al.
}

\author{
Sang Woon Kim • Sang Hyun Jee • Soo Young Moon • \\ Ji Yu Kim • Jang Hwan Kim
}

Published online: 22 October 2014

(C) The International Urogynecological Association 2014

We thank Agilli et al. [1] for their discussion of the restricted exclusion criteria in our study and possible effects of systemic conditions, including neuropsychiatric diseases and diabetes, on urinary nerve growth factor (NGF) [2]. NGF messenger RNA (mRNA) expression and protein production was described in various cells, including smooth muscle cells, urothelial cells, mast cells, and adipocytes. Raised circulating levels of NGF were known to be associated with several inflammation-related diseases, including metabolic syndrome, diabetes, and overactive bladder (OAB) [3].

As Agilli et al. pointed out, several medical conditions, including neuropsychiatric diseases, diabetes, and metabolic disorders, could affect the level of NGF. However, to our knowledge, most reported evidence involved serum or tissue, not urinary, NGF. Furthermore, some reports were demonstrated only in experimental animal studies. Also, association between medications, such as corticosteroids and herbal formulas and NGF level, was demonstrated in serum in a limited number of studies [4].

Serum NGF may affect urinary NGF level via renal secretion, the proportion to which is unknown. There is limited regarding correlation between serum and urinary NGF levels. Liu et al. reported that serum NGF and urinary NGF/creatinine levels increased in patients with overactive bladder (OAB) refractory to antimuscarinics and remained high after another 3 months of antimuscarinic therapy, suggesting an increased circulating NGF is associated with refractory OAB [5]. However, another study found no relationship between serum and urinary NGF levels in bladder pain syndrome/interstitial cystitis (BPS/IC) patients and between serum NGF levels and medical diseases, including diabetes and dyslipidemia [6].

Questions remain regarding these particular points. Although exclusion criteria should be established more specifically to emphasize bladder-related symptoms in a future study, based on current evidence, it is considered that our study was performed using appropriate exclusion criteria.

\section{References}

1. Agilli M, Aydin FN, Kurt YG, Cayci T (2014) Urinary nerve growth 53 factor correlates with the severity of urgency and pain: methodological evaluation. Int Urogynecol J. doi:10.1007/s00192-014-2532-5

2. Kim SW, Im YJ, Choi HC, Kang HJ, Kim JY, Kim JH (2014) Urinary nerve growth 53 factor correlates with the severity of urgency and pain. Int Urogynecol J. doi:10.1007/s00192-014-2424-8

3. Chaldakov GN, Fiore M, Stankulov IS, Manni L, Hristova MG, Antonelli A, Ghenev PI, Aloe L (2004) Neurotrophin presence in human coronary atherosclerosis and metabolic syndrome: a role for NGF and BDNF in cardiovascular disease? Prog Brain Res 146:279289. doi:10.1016/S0079-6123(03)46018-4

4. Mocchetti I, Spiga G, Hayes VY, Isackson PJ, Colangelo A (1996) Glucocorticoids differentially increase nerve growth factor and basic fibroblast growth factor expression in the rat brain. J Neurosci Off $\mathrm{J}$ Soc Neurosci 16(6):2141-2148

5. Liu HT, Lin H, Kuo HC (2011) Increased serum nerve growth factor levels in patients with overactive bladder syndrome refractory to antimuscarinic therapy. Neurourol Urodyn 30(8):1525-1529. doi:10. 1002/nau.21118

6. Liu HT, Kuo HC (2012) Increased urine and serum nerve growth factor levels in interstitial cystitis suggest chronic inflammation is involved in the pathogenesis of disease. PLoS One 7(9):e44687. doi: 10.1371/journal.pone.0044687
S. W. Kim • S. H. Jee · S. Y. Moon · J. Y. Kim • J. H. Kim $(\bowtie)$ Department of Urology, Urological Science Institute, Yonsei University College of Medicine, 50 Yonsei-ro, Seodaemun-gu, Seoul 120-752, South Korea

e-mail: JKIM@yuhs.ac 